\title{
A Study on Estimating the Elements of Card Game Fun Using Biological Information
}

\author{
Yuki Takaoka', Takashi Kawakami², Ryosuke 0oe ${ }^{2}$ \\ ${ }^{1}$ The Graduate School of Engineering, Hokkaido University of Science, Sapporo, Japan \\ ${ }^{2}$ Computer Science, Hokkaido University of Science, Sapporo, Japan \\ Email:9172001@hus.ac.jp, kawakami@hus.ac.jp, ooe-r@hus.ac.jp
}

How to cite this paper: Takaoka, Y., Kawakami, T. and Ooe, R. (2019) A Study on Estimating the Elements of Card Game Fun Using Biological Information. Journal of Computer and Communications, 7, 129-135. https://doi.org/10.4236/jcc.2019.712012

Received: October 22, 2019

Accepted: December 24, 2019

Published: December 27, 2019

\begin{abstract}
In this study, we conduct research to estimate the elements of fun in card games. Previously, we tried to estimate the elements of fun by conducting a questionnaire to players, but the results were not good. Therefore, we propose an analysis using the player's biological information to make a more accurate estimation. Specifically, we try to elucidate the elements of fun by having a player who is playing a game wear a smart watch, and measuring and analyzing the heart rate of that player. This paper conducts an experiment to determine whether our intended data can be collected. As a result, it was found that there is a response to the heart rate in a specific scene, and there is a possibility that the intended data can be collected. We plan to conduct larger experiments in the future.
\end{abstract}

\section{Keywords}

Definition of Fun, Biological Information, Game AI

\section{Introduction}

Game AI has been studied for a long time, and various AIs have been researched and developed. In recent years, the performance of computers has improved, and AI for games that are difficult to develop has also been studied. Examples are werewolf [1] and Texas Hold'em [2]. Especially in Texas Hold'em, AI is defeating top players. For these reasons, there is now an environment in which advanced AI can be developed. We also tried to develop AI for Hanafuda, a Japanese card game. Hanafuda is an imperfect information game and falls into a difficult to create. This is because it is difficult to design an evaluation function for evaluating the board and hand. To solve this problem, we proposed to apply UCT [3] used in Go [4] and Shogi [5] AI. As a result, AI ability was improved. 
However, AI that was too strong was not interesting. A player who played a game with AI that is too strong feels boring, and it seems that no one wants to try again. Therefore, we thought about creating an AI that would entertain human players. For that purpose, it is necessary to clarify fun of behaviors and elements in the game. We decided to use biological information as a means of elucidation. Specifically, the smart watch is put on the experimenter, and the heart rate while playing the Hanafuda is measured and analyzed. We investigate the situation in which the heart rate of the experimenter is responsive and propose that the elements appearing there are considered interesting. In this research, we will conduct experiments to collect what kind of reactions occurs during the Hanafuda as a basic research. This is because it is necessary to determine whether useful data can be acquired before conducting large-scale experiments. After confirming that useful data can be obtained, we will conduct extensive experiments.

The structure of this paper is as follows. First, the research done before is described, and the results and problems obtained are shown. After that, the result of the experiment conducted in this research and the direction for the future research are shown.

\section{Related Works}

In recent years, game AI that is not only strengthening game AI but also trying to entertain players is appearing. This chapter describes these AI examples and our research.

\subsection{Analysis of Fun in Soccer Games}

Takahashi et al. analyze scenes that human players find interesting in soccer games [6]. This research was done to build an interesting AI for soccer games. In this paper, an experiment was conducted to measure the fun using NPCs with interesting behaviors that they devised. A soccer simulator is used for the experiment, and the experimenter operates one person in the team. The remaining 10 people are programmed by the computer to take actions according to the fun they defined. It was done in a way that human players to evaluate whether the movements of computer players were interesting. As a result of experiments, it was found that human players prefer intelligent agents. In the future, they will investigate more effective actions and consider ways to take actions that suit human players' preferences.

\subsection{Go AI to Entertain Players}

There is a movement to entertain human player in Go. Ikeda et al. have reported preliminary experiments aimed at enumerating the elements to entertain human players [7]. The purpose of this paper is to express the fun using Monte Carlo Tree Search. As a matter, necessary for that:

1) Eliminate unnatural choice of AI 


\section{2) Produce various strategies}

An approach to solve these two points is shown. For the element of 1, they propose to improve the Monte Carlo method. The improvement is to intentionally select weak hands under specific conditions. By doing this, they built a player who was not too strong. When this method was evaluated, it was said that the weakness matched to the other party could be expressed. When this method was evaluated, they reported that they were able to express the weakness tailored to the human player. In the future, they will make evaluations by releasing the created programs and conducting large-scale experiments.

\subsection{Our Research}

Previously, we conducted research to construct AI for Hanafuda, which gives fun to human players [8]. The experiment was to create and evaluate a computer player that behaves based on the definition of fun that we defined. The evaluation method was performed by battling on a computer player and a human player and asking the human player about their impressions. The fun defined in this experiment is as follows:

1) Increase the variance of get or lose scores.

2) Adjust the final score of one match to near \pm 0 .

The first definition is to avoid boring matches. When there is little exchange of scores, it tends to be a boring game. This definition was adopted on the assumption that the impression of the match would be flashy and enjoyable if the big score moved. The second definition is to improve the impression of the match. If a human player experiences a big loss, the player will not want to play again. On the other hand, it seems that it is not interesting if the computer player is openly entertaining. Therefore, we decided it would be good if we could avoid both big wins and big losses and adjust the final score to around zero.

We created a computer player that satisfies these definitions and conducted a battle experiment with a human player. The results are shown in Table 1.

Analysis of these comments is as follows:

- It is not so strong that human player cannot win.

- Computer player is trying to entertain them.

- It feels too weak for some people.

- There are scenes in which selection is considered strange.

From these facts, the fun we have defined and method are not sufficient. Therefore, this study is conducted to develop a more accurate definition.

Table 1. Examples of winning hands of Koi-Koi.

\begin{tabular}{|c|c|c|}
\hline Name & Good point & Bad point \\
\hline $\mathrm{A}$ & $\begin{array}{l}\text { - It is not too strong, but it will } \\
\text { not let me win easily. } \\
\text { Not a one-sided match, I can enjoy } \\
\text { a match to a certain extent. }\end{array}$ & $\begin{array}{l}\text { Computer players sometimes } \\
\text { do strange selection. }\end{array}$ \\
\hline B & - I could see it trying to make a game. & $\begin{array}{l}\text { - The strength was not constant. } \\
\text { I felt it was often too weak. }\end{array}$ \\
\hline
\end{tabular}




\section{Experiment}

In this study, we conducted an experiment to measure and analyze the heart rate of a human player. Humans experience psychological changes when they are in a critical phase, such as when they are tense or thinking. By measuring this change, we can observe that this situation is serious for the player. Therefore, we hypothesized that a situation that causes some characteristic change is an element of fun. This experiment is based on this hypothesis. In this paper, the explanation of the Hanafuda is omitted. Note that see [8] for rules of Hanafuda.

\subsection{Equipment Used}

A smartwatch called Fitbit Charge 2 is used to measure the heart rate. This device can acquire heart rate in 1 second unit, and it is enough to measure the change in the match. The measured data is saved on the server and recorded along with the time. Data can be extracted from the python API, making analysis easier. These are the reasons for selecting Fitbit Charge 2.

\subsection{Experimental Method}

The experiment is performed by wearing a smart watch on the subject and playing the Hanafuda while measuring the heart rate. At the same time, the play screen is recorded so that the relationship between the game situation and heart rate can be verified. Cross-reference the recorded data and heart rate data to find a characteristic situation. The battle is performed with software built in AI constructed in [8]. Figure 1 shows this actual screen. This software has a GUI, and to play the game by clicking on the card. In this experiment, a touch panel was adopted so that the subject could proceed by touching the card. With this method, we thought that it would be possible to obtain more realistic heart rate changes without having to move the mouse to click.

Experiment is done by player accustomed to Hanafuda. Data is collected as much as possible without setting the number of times. Then, observe and analyze what kind of data was collected.

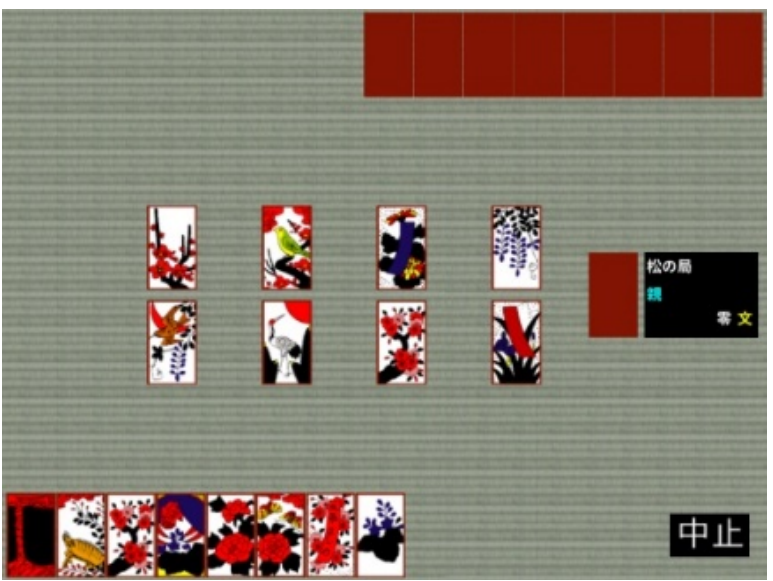

Figure 1. Experimental game screen. 


\subsection{Experimental Result and Discussion}

This section describes data that was particularly characteristic. Figure 2 shows a specific situation during the game, and Figure 3 shows some of the changes in heart rate for the match in Figure 2. The black circle in Figure 3 is the heart rate recorded at the time of Figure 2. The vertical axis in Figure 3 is the measured heart rate, and the horizontal axis is the passage of time. Indicating that the game is in progress as it goes to the right of the figure. The situation in Figure 2 is as follows:

- The human player is aiming for Hanami-Zake.

- The computer player aims for Hikari.

- There is the Sakura Curtain in the human player's hand.

- There is no 3rd month card in the field.

Under this situation, it can be said that the human player can win or lose the match based on if the Sakura Curtain card can be obtained. As Figure 3 shows, the heart rate increases as the game progresses. Eventually the peak was when both players ran out of cards and the game ended. From this, it is considered that the human player was in a state of tension, thinking "Can I get this card?" It can also be read from the heart rate increases as the game progresses. Therefore, the elements of the situation in Figure 2 may be fun. Analyzing the elements of

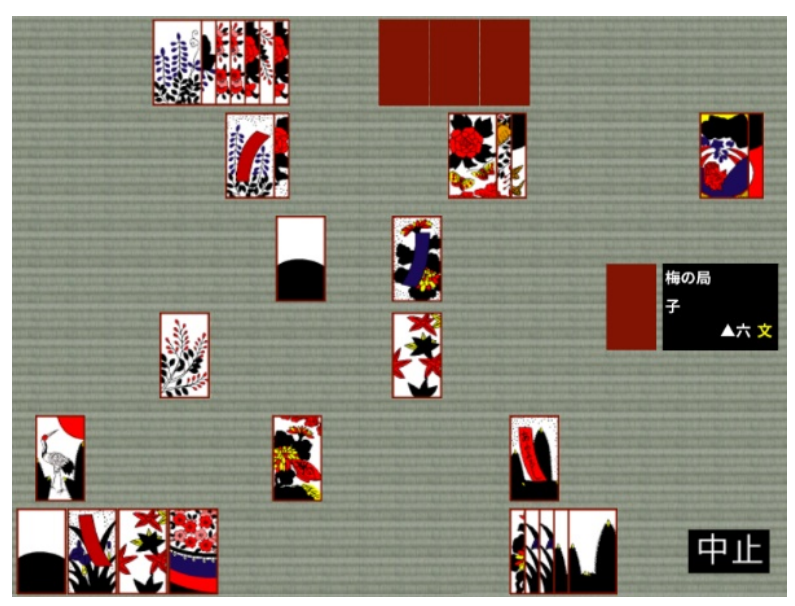

Figure 2. A specific situation during the game.

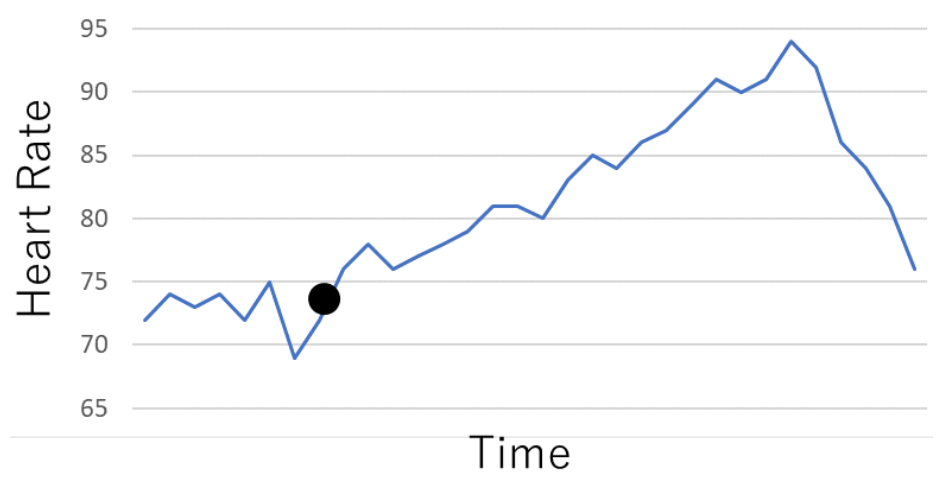

Figure 3. Changes in human player's heart rate. 
the situation in Figure 2, the element "whether or not the card can be taken" emerges. "Whether or not card can be taken" is often seen in real match. Furthermore, assuming that there are spectators in the Hanafuda, "whether or not the card can be taken" is an easy-to-understand element. Therefore, there is a possibility that the element "Whether or not card can be taken" is one of the fun elements of the Hanafuda.

In this study, several data were collected in addition to the exemplified data. Therefore, our method proved effective. In the future, more experiments will be repeated, and data will be collected and analyzed.

\section{Conclusions}

In this study, we conducted an experiment using heart rate to analyze the fun elements of Hanafuda. As an experimental method, we planned to measure the heart rate with a smartwatch while playing the Hanafuda and then analyze it. As a result, it was confirmed that there was a situation where the heart rate changed. Therefore, it was proved that the method we examined was valid.

The following two points are given as future research:

1) Increase the number of data.

2) Utilization of diverse biological information.

Especially for 2. it is expected that more accurate analysis will be possible. Currently, only heart rate is used, but there are other types of biological information. Combining multiple pieces of biological information may lead to improved reliability. In addition, it is necessary to examine the selection of measuring instruments from a medical point of view. This is because reliable equipment is required for correct analysis. We would like to investigate these issues and proceed with our research in the future.

\section{Conflicts of Interest}

The authors declare no conflicts of interest regarding the publication of this paper.

\section{References}

[1] Zhang, M. (2019) Solving the Werewolf Puzzle by Communication between Agents [Project Paper]. http://hdl.handle.net/10119/15900

[2] Brown, N. and Sandholm, T. (2019) Superhuman AI for Multiplayer Poker. Science, 365. https://doi.org/10.1126/science.aay 2400

[3] Auer, P., Ceca-Bianchi, N. and Fischer, P. (2002) Finite-Time Analysis of the Multiarmed Bandit Problem. Machine Learning, 47, 235-256. https://doi.org/10.1023/A:1013689704352

[4] Gelly, S., Wang, Y., Munos, R. and Taytaud, O. (2006) Modification of UCT with Patterns in Monte-Carlo Go. Technical Report RR-6062.

[5] Sato, Y. and Takahashi, D. (2009) A Shogi Program Based on Monte-Carlo Tree Search. IPSJ Journal, 50, 2740-2751.

[6] Takahashi, K. and Nishino, J. (2017) Entertainer NPC's Action on Soccer Game. 
Proceedings of the Fuzzy System Symposium, Yamagata, 13-15 September 2017, 575-578.

[7] Kokolo, I. and Viennot, S. (2012) Production of Various Strategies and Position Control for Monte-Carlo Go-Entertaining Human Players. Game Programming Workshop 2012, Kanagawa, 9-11 November 2012, 47-54.

http://hdl.handle.net/10119/11605

[8] Takaoka, Y., Kawakami, T. and Ooe, R. (2018) A Fundamental Study of a Computer Player Giving Fun to the Opponent. Computer Science \& Communications, 6, 32-41. https://doi.org/10.4236/jcc.2018.61004 